\title{
THE 0.3 TO 100 MICRON CONTINUA OF TYPE 1 SEYFERTS
}

\author{
Martin J. Ward \\ Institute of Astronomy, Madingley Rd. Cambridge CB3 OHA, UK \\ and. Dept. of Astronomy FM-20, University of Washington \\ Seattle WA 98195, USA \\ N.P. Carleton, M. Elvis, G. Fabbiano, S.P. Willner \\ Center for Astrophysics, 60 Garden Street \\ Cambridge MA 02138, USA
}

\begin{abstract}
A. Lawrence
Mathematics Dept. Queen Mary College, Mile End Road

London E1, UK
\end{abstract}

\begin{abstract}
The continua of type 1 Seyferts from 0.3 to 100 microns have been examined. We focus on the modifying effects of dust, both in terms of absorption and re-radiation. After allowance is made for dust and the presence of extranuclear contributions to the IRAS fluxes, there remains a ubiquitous underlying distribution which we believe to be non-thermal. There is evidence that the thermal infrared components corresponding to hot and warm dust may be associated with the broad and narrow emission line regions.
\end{abstract}

\section{INTRODUCTION}

A long standing controversy concerning the infrared continuum emission from Seyfert 1s has centered on whether it is predominantly thermal or non-thermal in origin. A related question is whether the optical and infrared continua have distinct origins. There is increasing evidence that this is so. There is a general lack of fast variability of the infrared component in contrast to the commonly variable optical and ultraviolet regions. Also. the continua often change slope around 1 micron, suggesting that a new component is dominating the infrared. This paper presents the evidence in favor of the hypothesis that dust plays an important role in modifying the optical to infrared continuum. For the sake of brevity an extensive discussion of the observations and statistical tests is not given here. A more detailed description of these can be found in Ward et al. (1987) and Carleton et al. (1987).

\section{SELECTION EFFECTS - IMPORTA.NCE OF AN UNBIASED SAMPLE}

Samples of AGN have been selected from surveys at radio, infrared, blueoptical and X-ray frequencies (see the review by Cannon in these proceedings). 
We are concerned with the effects of dust in "typical" type 1 Seyferts. Therefore, it is desirable to use as our sample one which is not prone to preferentially select examples of highly reddened objects. Conversely, we wish to avoid samples which select Seyferts only if they are subject to little or no reddening. The Markarian Seyferts would generally fall into the latter category, and probably IRAS selected AGN the former category, although the dust properties of the IRAS selected Seyfert $1 \mathrm{~s}$ is still a matter for study. The sample used in this work contains AGN identified from the hard X-ray catalog of Piccinotti et al. (1982). This sample should not be biased either in favor or against the presence of dust, since the hard $\mathrm{X}$-rays are not significantly attenuated by the equivalent column density of many magnitudes of visual obscuration. Both because the AGN in the Piccinotti et al. sample are relatively bright and thus well studied, and as a result of new midinfrared observations (Ward et al. and Carleton et al.), there are extensive data available on their continua and emission line fluxes. For most objects we have continuum fluxes from 0.3 microns (U-band), to 100 microns (IRAS Point source catalog 1985).

\section{THE ENERGY DISTRIBUTIONS}

\subsection{A Morphological Classification}

To set the 0.3 to 100 micron continuum spectrum of AGN into context, we show in Figure 1 the radio through gamma ray distribution for $3 \mathrm{C} 273$.

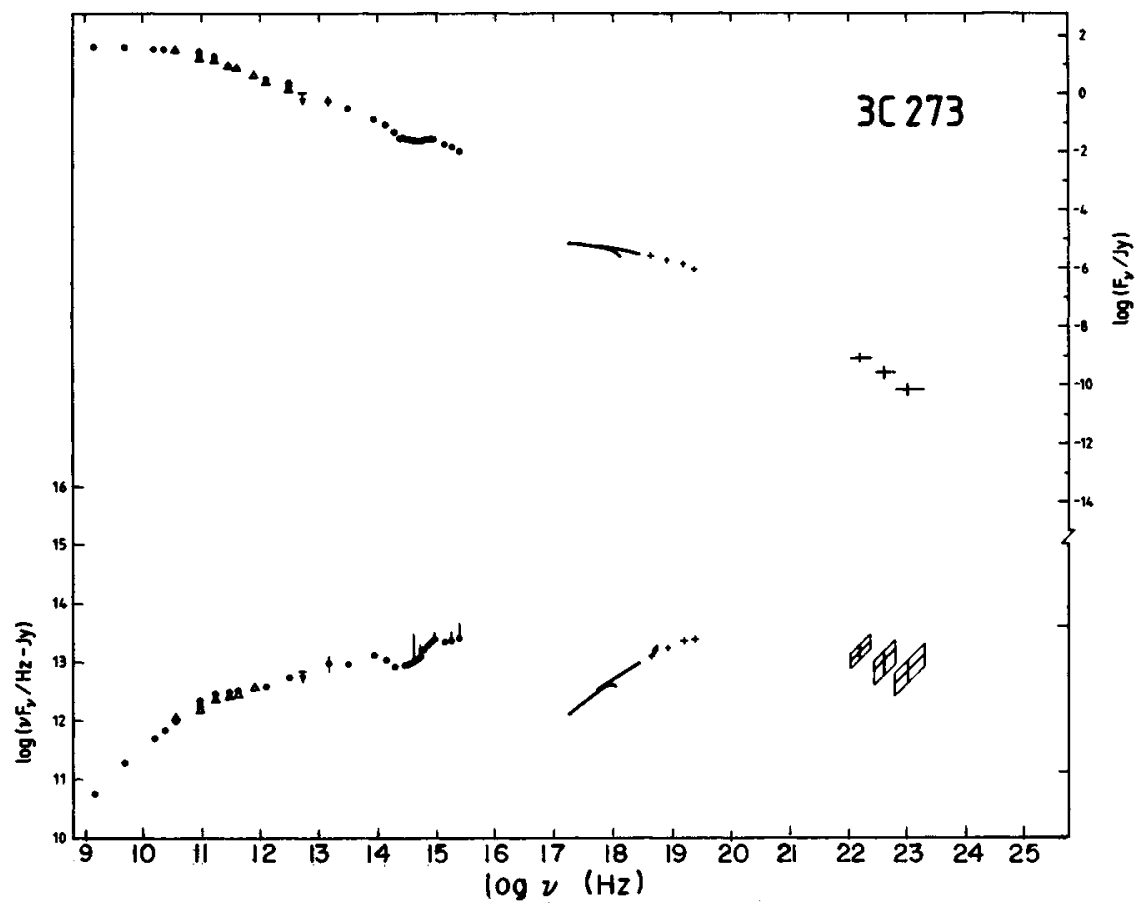

Figure 1. The radio to gamma ray energy distribution of $3 \mathrm{C} 273$ (adapted from Perry, Ward and Jones 1986). 
Figure 1 displays two methods of representing the same data. The top plot is the standard $\log \mathrm{f}_{\nu}$ vs $\log \nu$, and the lower plot is the more physically instructive $\log \nu . f_{\nu}$ vs $\log \nu$, in which it can be easily seen where the maximum energy per frequency interval is emitted. We use the $\log \nu . f_{\nu}$ vs $\log \nu$ plot for our morphological classification of the energy distributions. Although $3 \mathrm{C} 273$ is atypical of the majority of our sample, in terms of its strong radio emission and high luminosity, it nevertheless exhibits important salient features of Seyfert 1s. In particular we note that in the lower plot the energy output does not span more than factor of 100 in amplitude from the radio to gamma ray frequencies. It is possible that peaks may be present in the unobserved extreme ultraviolet and very hard X-ray regions. It is also apparent that power laws fit the data only over rather limited frequency ranges. Although this is in part due to other components superimposed on an underlying continuum as we shall discuss below.

In our sample we identify three characteristic 0.3 to 100 micron distributions. Examples of these are shown in Figure 2.
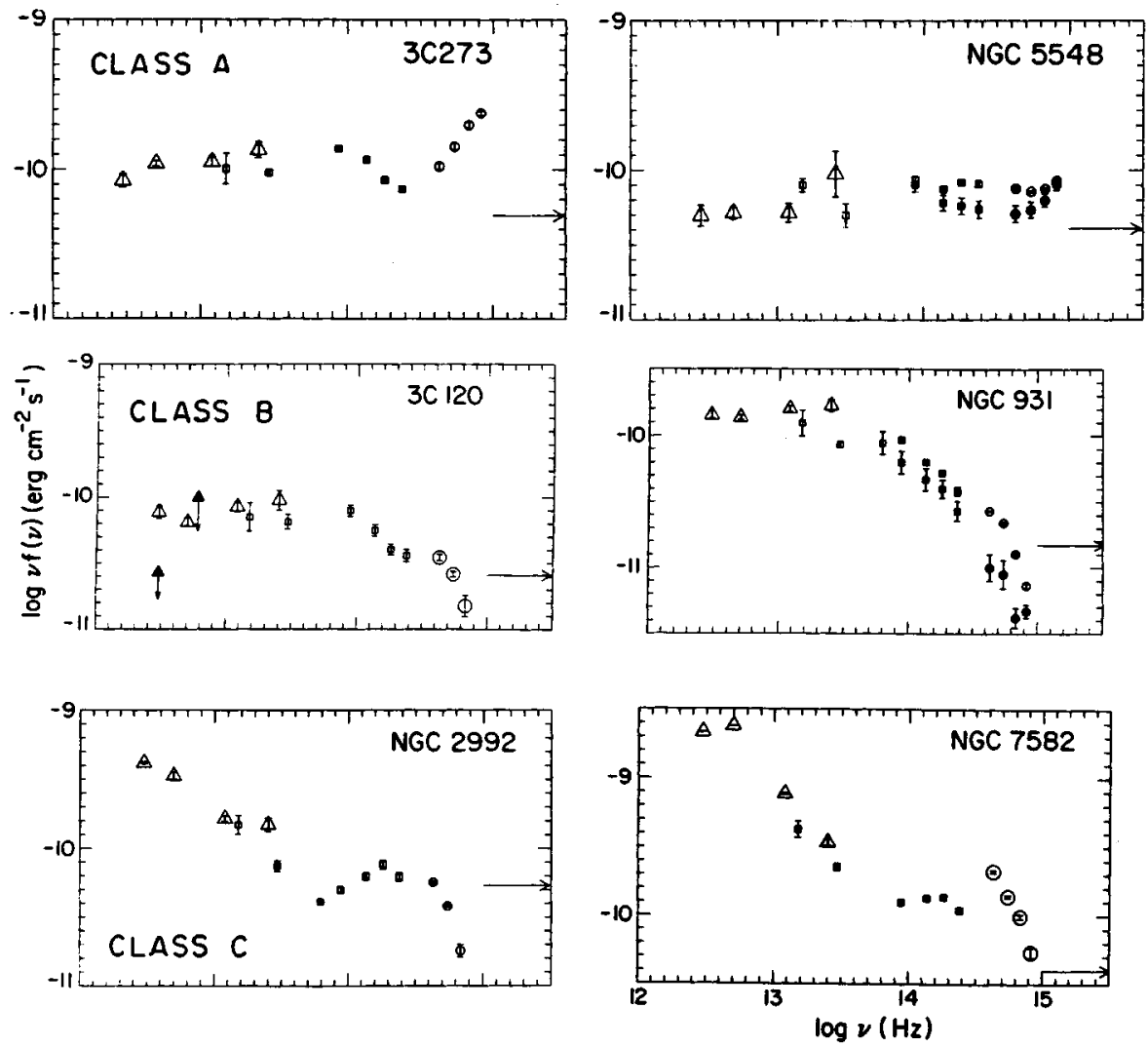

Figure 2. Two examples of each class of 0.3 to 100 micron energy distribution. 
For convenience we call the Seyferts with generally flat distributions but excesses in the blue optical regions, Class $\mathrm{A}$. Those with falling distributions toward the higher optical frequencies but fairly flat far-infrared spectra Class B, and those with steeply rising flux from 12 micron to 60 micron Class $C$. These classes may be clearly identified using the two-color diagram shown in Figure 3. The "colors" are the $\log \nu \cdot f_{\nu}$ flux ratios at 1.2 and .36 microns and the IRAS measured fluxes at 60 and 12 microns. Note the absence of objects with strong blue excesses and steeply rising IRAS flux distributions. We shall propose an explanation for this below.

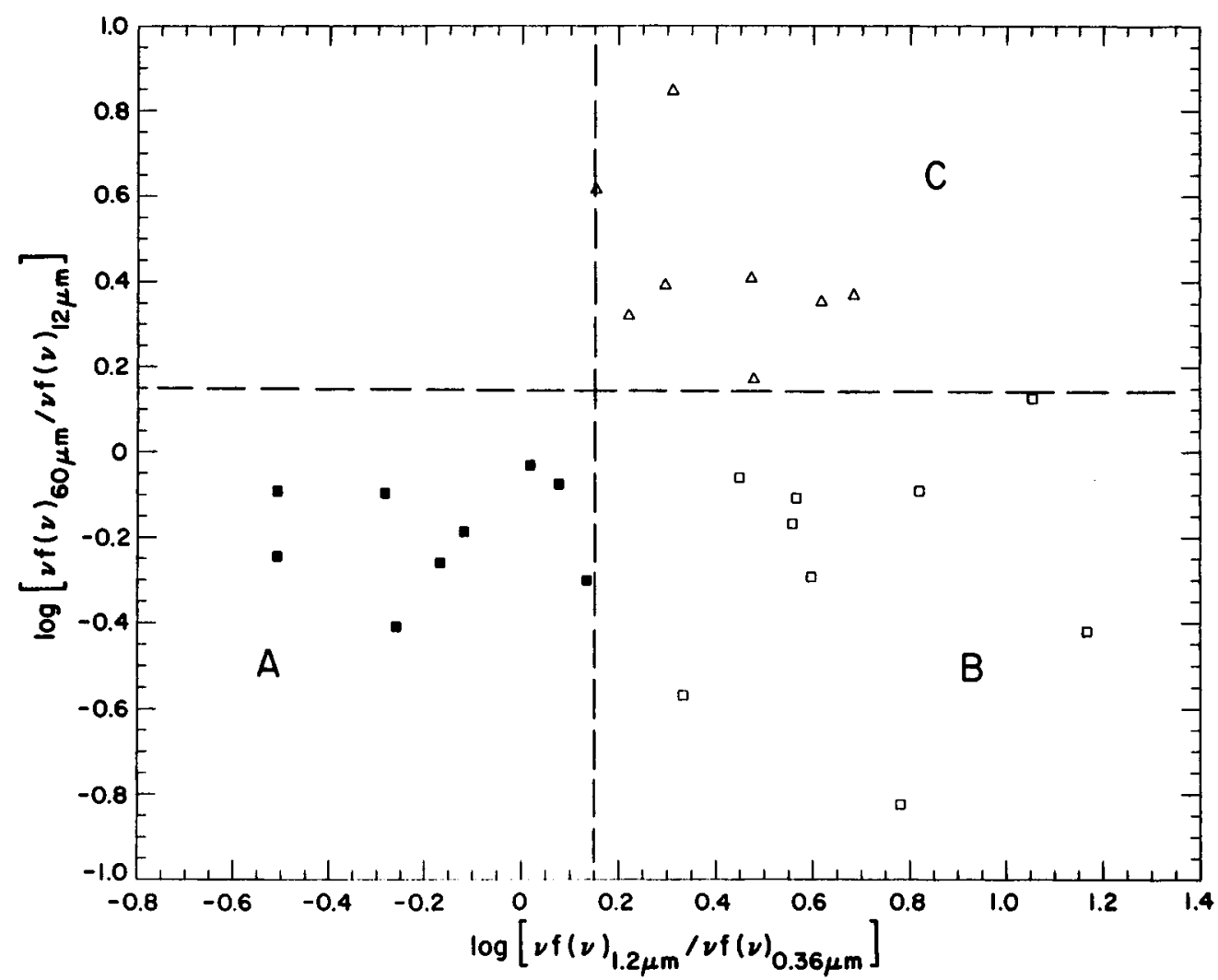

Figure 3. Flux ratio diagram, distinguishing between the 3 classes of energy distribution.

\subsection{A Similar Underlying Continuum?}

Despite the apparently large differences in the 0.3 to 100 micron distributions shown in Figure 2, we suggest that there is an underlying simplicity. Class A represents the nearest approximation to an AGN free of reddening and contamination from the underlying galaxy. Class $B$ represents a reddened AGN which is otherwise the same as Class $\mathrm{A}$, and Class $\mathrm{C}$ objects have infrared fluxes that are dominated by components outside the compact nucleus, either a circum- nuclear 
starburst or emission from the underlying galaxy. These assertions are supported by a substantial amount of statistical evidence. For example, the Class B objects are found to be reddened by from 1 to 3 visual magnitudes, based on both their Balmer decrements and the ratios of $\mathrm{H} \alpha$ to hard $\mathrm{X}$-ray flux. Using the same criteria the reddening in Class $A$ objects is found to be relatively small. The Class $C$ Seyferts usually have significant stellar contamination in the optical and near infrared, and some exhibit features in their 8-13 micron spectra indicating the presence of a starburst component. The absence of objects in the top left of Figure 3 suggests that those galaxies with blue optical continua and little reddening lack the thermal components in the far infrared that we propose to be of extra-nuclear origin. What is the slope of this underlying continuum? In order to avoid the problem of contamination by the Balmer continuum and the onset of the so-called "big blue bump", the postulated 25,000 - 30,000 $\mathrm{K}$ accretion disc (Malkan and Sargent 1982), we have fitted the continua from 1 to 100 microns. For the Class A objects we find a surprisingly unique value of $\alpha$ (ir) $=-1.02 \pm .10$. We next examine the connection, if any, between this and the $\mathrm{X}$-ray region. The power law indices that connect the 1.2 micron end of the baseline with the hard X-ray point at $6 \mathrm{keV}$ have an average value of $\alpha(\mathrm{ir} / \mathrm{X}-\mathrm{ray})=-1.05 \pm .05$. The similarity of this value to the infrared index suggests that there may be an underlying power law of slope -1 which extends from 100 microns to $6 \mathrm{keV}$. However, the slopes measured in the $\mathrm{X}$-ray region for these objects are typically -0.7 (Petre et al. 1984). so the assumption of a single power law must be an oversimplification. The $\mathrm{X}$-ray flux could be a composite of Compton emission and the power law seen at longer wavelengths.

\section{THE RELATIVE THERMAL AND NON-THERMAL CONTRIBUTIONS}

\subsection{The Non-Thermal Component}

It is reasonable to assume that the dust which absorbs a part of the opticalultraviolet continuum also contributes a portion of the 1 to 100 micron continuum by virtue of re-radiation. Since this thermal contribution will differ in each case it would be most useful to be able to quantify the effect. We can calculate the infrared luminosity of the power law component by assuming that the index $\alpha=-1$ and identifying the lowest single point of each spectrum between 1 and 100 microns. This lowest continuum point is assumed to be least contaminated by thermal emission. The wavelength of the low point will differ from object to object since the dust grain temperature distributions will not be unique. The area under this baseline is taken to represent the non-thermal luminosity, whilst the area above is the thermal component. We find that in Class $\mathbf{A}$ objects on average the thermal component is $70 \%$ of the non-thermal, whereas for Class B objects the thermal emission is typically twice as strong as the non-thermal flux, consistent with our hypothesis concerning the relative importance of dust. Support for our method of de-convolving the infrared components is found in an X-ray vs. infrared correlation. It has long been established that the 3.5 micron and hard X-ray luminosities are particularly well correlated (Malken 1984). However, the same analysis using our power-law infrared luminosity in place of that at 3.5 microns, yields a significantly improved correlation with the $\mathrm{X}$-rays. 


\subsection{The Nature of the Thermal Components}

The wavelength at which the thermal emission peaks will depend on the detailed temperature distribution of the dust grains. It is possible to make simple calculations to derive the distance of a dust grain of a given temperature from a heating source of given luminosity, for example see Davidson and Netzer (1979). For average values of the luminosity we find that hot dust $(1000 \mathrm{~K})$ emits at a radius of about $0.7 \mathrm{pc}$ and warm dust $(180 \mathrm{~K})$ at a radius of $50 \mathrm{pc}$. In view of the simplicity of the model these must be considered only rough numbers, but the near coincidence of sizes compared to the broad and narrow emission line regions is interesting. If the hot and warm dust is indeed spatially associated with the emission line regions then one might expect a close connection between $\mathrm{H} \alpha$ and 3.5 micron emission, and between the [0III] luminosity and that at 20 microns. This is found to be the case. The 3.5 micron luminosity correlates better with $\mathrm{L}(\mathrm{H}$ $\alpha$ ) than with $\mathrm{L}([\mathrm{OIII}])$, while the $\mathrm{L}(20$ micron) correlates better with $\mathrm{L}([\mathrm{OIII}])$. Another way to probe the dust and gas properties is to look at the emission line and continuum ratios. As the narrow line region becomes more dominant with respect to the broad line region i.e., high $[0 \mathrm{III}] / \mathrm{H} \beta$, so the cooler dust emission grows relative to the hot dust emission i.e., high 20 micron $/ 3.5$ micron, (the ir fluxes used in the ratio are obtained after the subtraction of the underlying power law component).

\section{THE ORIGIN OF INFRARED CONTINUUM - FURTHER TESTS}

Although our hypothesis concerning the importance of dust and its relative contribution to the infrared continuum is consistent with present data we would naturally like to test its validity further. There are several ways this may be done in the near future.

Infrared monitoring at 10 and 20 microns is now feasible with accuracies of between 10 and $20 \%$. In the luminosity range of our sample short-term variations of from days to weeks would be difficult to accommodate in a predominately thermal model. Unfortunately, long-term changes over months to years can occur in both thermal and non-thermal components..

We have mentioned that the 8 - 13 micron spectra of some AGN contain dust signatures. The starburst nuclei have strong features at 11.25 and 8.65 microns and the $[\mathrm{NeII}]$ fine structure line at 12.8 microns. In contrast, Seyferts generally have a featureless spectra, Aitken and Roche (1985). These starburst features probably arise when small grains suffer thermal "spikes" after absorption of ultraviolet photons (Sellgren 1984). In Seyfert nuclei these tiny grains may be evaporated by the harder ultraviolet continuum, but the larger grains survive and re-radiate. $\mathbf{A}$ range of temperatures could explain the smooth 8-13 micron spectra characteristic of Seyferts. Thus, the presence of features (e.g., NGC 7469 and NGC 7582 Figure 4) exposes the existence of a nuclear starburst component in a few Seyferts, and implies significant thermal infrared emission in these cases, but the absence of such features in the majority does not prove the non-thermal case.

Another line of attack would be to extend the continuum coverage to the $\mathrm{mm}$. and sub-mm. region. Unfortunately, with present sensitivities at these frequencies it is extremely difficult to distinguish between a thermal Rayleigh-Jeans and a nonthermal component turn-over beginning near, or beyond, 100 microns. 
Perhaps one of the most promising ways of testing the suggestion that the NLR gas may be co-spatial with a warm $(200 \mathrm{~K})$ dust component, is to image the 20 micron emission. For the nearer Seyferts the NLR extends over many arcseconds and the newly available infrared imaging arrays with pixel sizes less than 1 arcsecond are thus well matched for studies on these scales. They will permit us to directly compare the infrared and emission line region morphologies.
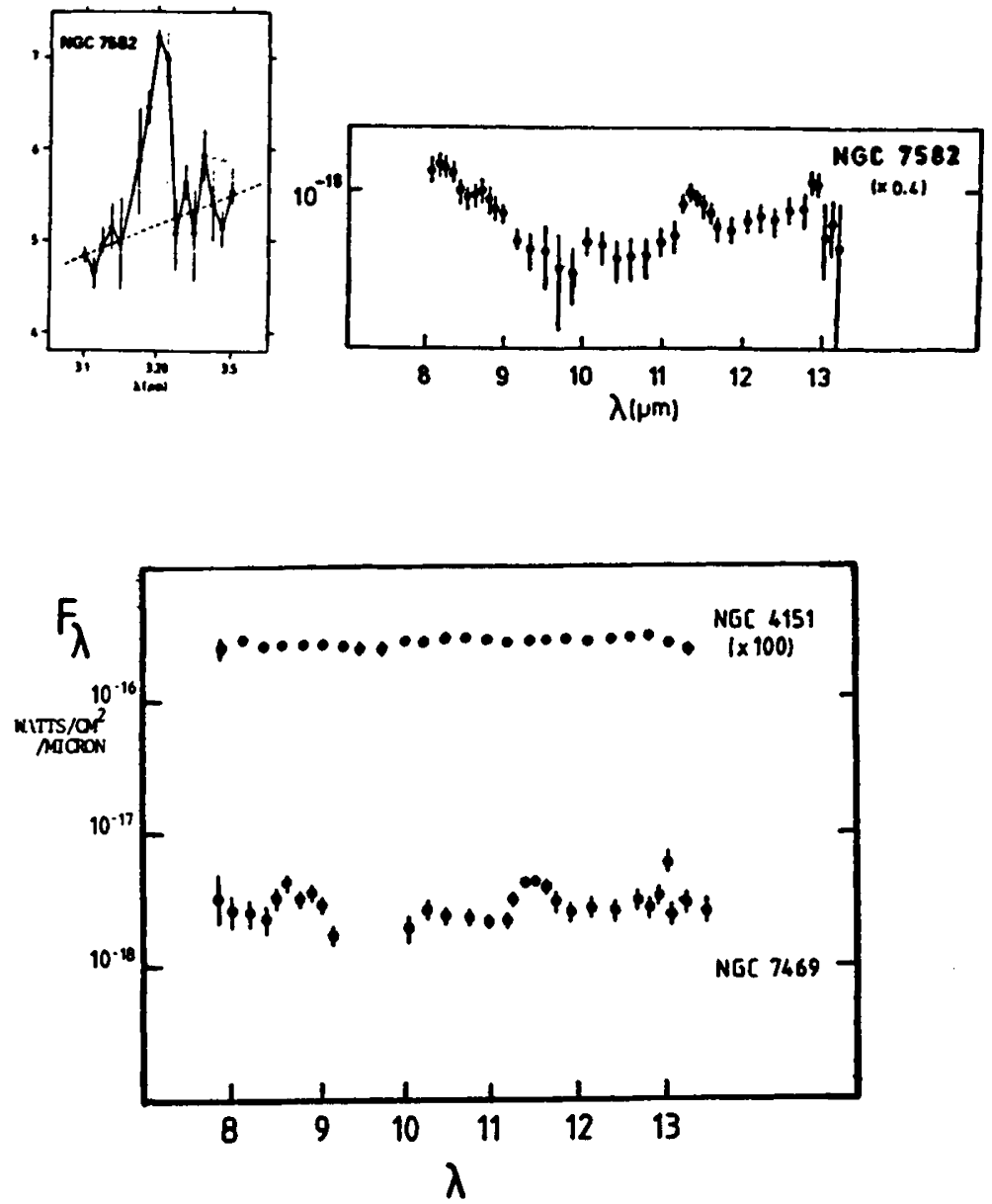

Figure 4. Top, dust related features in the narrow line X-ray emitting galaxy NGC 7582. From Moorwood ESO Messenger No. 27, page 11, and Roche et al. 1984.

Bottom, a typical featureless Seyfert spectrum NGC 4151 and the unusual case of the Seyfert NGC 7469, Aitken et al. 1981. 


\section{References}

Aitken, D., and Roche, P.F. 1985, MNRAS, 213, 777.

Aitken, D., Roche, P.F. and Phillips, M.M. 1981, MNRAS, 196, $101 \mathrm{P}$.

Carleton, N.P., Elvis, M., Fabbiano, S.P., Willner, S.P., Lawrence, A and Ward, M.J. 1987, Ap. J., submitted.

Davidson, K. and Netzer, H. 1979, Rev. Mod. Phys., 51, 715.

Infrared Astronomical Satellite (IRAS) : The Point Source Catalog 1985, (Washington: Government Printing Office).

Malkan, M.A. and Sargent, W.L.W. 1982, Ap. J., 254, 22.

Malkan, M.A. 1984, in X-ray and UV Emission from Active Galactic Nuclei, eds. W. Brinkmann and S. Trümper (Max-Planck-Institut für Extraterrestriche Physik) p.121

Perry, J., Ward, M.J. and Jones, M. 1986, preprint.

Petre, R., Mushotzky, R.F., Krolik, J.H. and Holt, S.S. 1984, Ap. J., $280,499$.

Piccinotti, G., Mushotsky, R. E., Bolt, E. A., Holt, S.S., Marshall, E.E., Serlemitsos, P.J., and Shafer, R.A. 1982, Ap. J., 253, 485.

Roche, P.F., Aitken, D.K., Phillips, M.M., Whitmore, B. 1984, MNRAS, 207, 35.

Sellgren, K. 1984, Ap. J., 277, 623.

Ward, M.J., Elvis, M., Fabbiano, G., Carleton, N.P., Willner, S.P and Lawrence, A. 1987, Ap. J., accepted.

\section{DISCUSSION}

ANTONUCCI: In the figure you showed you tied down the long wavelength end of your power law at $100 \mu$, but radio quiet objects are declining between $100 \mu$ and $1 \mathrm{mrn}$. Therefore your long wavelength "low point" would have been very different had the IRAS data extended to longer wavelength.

WARD: You are correct that some of our continua distributions have turned over at 100 microns. These are often the objects which we have called "CLASS B", that is to say they would be like "CLASS A" but for absorption (optical) and re-radiation (infrared) due to the presence of dust. The range in our estimates of the power law index does indeed have largor scatter than for "CLASS A".

MACCHETTO: The potver law index as determined seems to depend heavily on the $100 \mu$ point. As you mentioned this can be too large by factors of 3-10. If this is so you would get too steep an index.

WARD: 0 if the 30 objects only a few have the "Low points" used to determine the power law at 100 microns. To turn the question around, The fact that the derived index has such a small range suggests that the extended component at 100 microns is not seriously contarninating the compact 100 inicron source. 